\title{
EL MODELO TPACK EN LA PRAXIS DOCENTE EN UNA UNIVERSIDAD ARGENTINA. CONOCIMIENTOS Y PRÁCTICAS DOCENTES EN TORNO AL CONOCIMIENTO DIDÁCTICO-TECNOLÓGICO DEL CONTENIDO (CDTC) EN AULAS UNIVERSITARIAS
}

\author{
Fernando A. Flores* \\ CONICET - Universidad Nacional del Nordeste, Argentina \\ fas_flores@yahoo.com.ar \\ Margarita C. Ortiz* \\ Universidad Nacional del Nordeste, Argentina \\ margaritacristinaortiz@gmail.com.ar
}

Recibido: 31/10/2018 Aceptado: 19/02/2019

\section{Resumen}

Presentamos una aproximación a los conocimientos y prácticas docentes en torno al conocimiento didáctico-tecnológico del contenido (CDTC) o Technological Pedagogical Content Knowledge (TPACK) en aulas universitarias de carreras humanísticas en una universidad argentina.

Metodológicamente, construimos un caso múltiple constituido por equipos docentes de seis asignaturas vinculadas con Tecnología educativa de Profesorados y Licenciaturas de una de las Unidades Académicas. También realizamos una doble triangulación: intra-metodológica (fuentes primarias y secundarias) y de investigadores y docentes participantes como estrategia de validación pertinente en un estudio cualitativo.

Encontramos que las maneras de abordar la relación teoría-práctica devienen en variadas configuraciones del CDTC, según las trayectorias profesionales de las profesoras y entramados de experiencias de formación permanente, gestión e investigación. Configuraciones construidas individualmente y re-construidas colectivamente en los territorios disciplinares-académicos y diferentes contextos.

Palabras clave: La dimensión tecnológica en el CDC - Integración curricular de las TIC - Tensión teoría-práctica - Docencia universitaria - Estudio de caso múltiple.

\footnotetext{
* Profesor Auxiliar de las asignaturas: Investigación Educativa I, Profesorado y Licenciatura en Ciencias de la Educación, Facultad de Humanidades - UNNE; y de Epistemología en Ciencias de la Cultura, Licenciatura en Gestión y Desarrollo Cultural, Facultad de Artes, Diseño y Ciencias de la Cultura - UNNE. Profesor y Licenciado en Ciencias de la Educación. Tesis de Maestría en evaluación, Maestría en Metodología de la Investigación Científica, Facultad de Humanidades-UNNE. Doctorando del Doctorado en Humanidades, Facultad de Filosofía y Letras, Universidad Nacional de Tucumán. Becario de finalización doctoral del CONICET. Realiza tareas de investigación en el Instituto de Investigaciones en Educación, Facultad de Humanidades, Universidad Nacional del Nordeste.

" Docente investigadora del Instituto de Investigaciones en Educación. Facultad de Humanidades. Universidad Nacional del Nordeste. Profesora Adjunta Ordinaria de la asignatura Metodología de la Investigación, Departamento de Educación Inicial. Jefe de Trabajos Prácticos de la asignatura Biología del Aprendizaje, Departamento de Ciencias de la Educación. Facultad de Humanidades, UNNE. Profesora en Biología. Especialista en Investigación Educativa (Universidad del Comahüe) Magíster en Epistemología y Metodología de la Investigación, Facultad de Humanidades, UNNE. Doctoranda del Doctorado en Ciencias Cognitivas, Facultad de Humanidades, UNNE. Co-directora del PI: "La construcción del conocimiento didáctico del contenido en profesores experimentados y principiantes de la Universidad Nacional del Nordeste. Estudio de casos múltiples", acreditado por la SGC y T de la UNNE.
} 
Revista de la Escuela de Ciencias de la Educación, año 15, nRo. 14, vol 2. Julio a Diciembre de 2019. Páginas 14-27. ISSN 18516297 (DESDE DICIEMBRE DE 2006 A DICIEMBRE DE 2017). ISSN 2362-3349 (EN LÍNEA). EL MODELO TPACK EN LA PRAXIS DOCENTE EN UNA UNIVERSIDAD ARGENTINA. CONOCIMIENTOS Y PRÁCTICAS DOCENTES EN TORNO AL CONOCIMIENTO DIDÁCTICO-TECNOLÓGICO DEL CONTENIDO (CDTC) en aulas universitarias. Fernando, A. Flores. Margarita, C. Ortiz.

\section{Abstract}

We present an approach to the Knowledge and teaching practices around the Technological Pedagogical Content Knowledge (TPACK) or Conocimiento Didáctico-Tecnológico del Contenido (CDTC) in university classrooms of humanistic careers in an Argentine university.

Methodologically, we built a multiple case consisting of teaching teams of six subjects linked to the Educational Technology of Teachers and Degrees from one of the Academic Units. Also, we perform a double triangulation: intra - methodological (primary and secondary sources) and of participating researchers and teachers as a relevant validation strategy in a qualitative study.

We find that the ways of approaching the theory-practice relationship come in various configurations of the TPACK, according to the professional trajectories of the teachers and networks of experiences of permanent training, management and research. Configurations built individually and collectively re-built in the disciplinary-academic territories and different contexts.

Key words: The technological dimension in the PCK - Curricular integration of ICT - Theorypractice tension - University teaching - Multiple case study.

\section{Introducción}

Presentamos algunos hallazgos respecto de las configuraciones que adopta el CDTC en las prácticas de enseñanza y aprendizaje presenciales y virtuales de profesoras de cátedras del área Tecnología Educativa de Profesorados y Licenciaturas diversos de una de las Facultades de una universidad pública del norte argentino. Se trata de un estudio en curso del Programa de Investigación "Conocimiento y Formación Docente" del Instituto de Investigaciones en Educación, de la unidad académica en la que se desempeñan estas docentes.

Empleamos sin distinción las siglas en español e inglés al referirnos al Conocimiento Didáctico del Contenido (CDC - PCK) y Conocimiento Didáctico Tecnológico el Contenido (CDTC-TPACK). Este constructo, propuesto por Mishra y Koehler (2006), amplió la noción del PCK de Shulman (1986) y muestra un incipiente desarrollo en la enseñanza superior.

Optamos por el estudio de casos como estrategia metodológica para abordar en profundidad el citado constructo. Al ser un estudio cualitativo, nos proponemos avanzar en la interpretación y comprensión del objeto de estudio, con el objetivo de examinar las subcategorías emergentes en una de las funciones del profesor universitario: la dimensión docente, a la luz del modelo TPACK,entramada con las de investigación y gestión en este ámbito.

Estructuramos el artículo en las secciones siguientes: en la primera, exponemos el encuadre referencial; en la segunda, las decisiones metodológicas adoptadas; en la siguiente, relatamos el caso colectivo incrustado; en la cuarta, comunicamos los resultados y en la última, las hipótesis interpretativas a modo de conclusiones.

\section{Encuadre teórico referencial}

Actualmente, existe un amplio acuerdo (Marcelo, Yot y Mayor, 2015; Cabero Almenara, Marín Díaz y Castaño Garrido, 2015; Cabero Almenara, 2014; Gewerc, Pernas y Varela; 2013) en considerar el impacto reciente de la dimensión tecnológica en el desarrollo y evolución del conocimiento profesional del docente (CPD), contextos socio-educativos en el que las TIC adquieren mayor protagonismo. En este sentido, desde el punto de vista didáctico, su utilización en la enseñanza universitaria implica la inclusión de un componente curricular que condiciona -y en ocasiones-, transforma el conocimiento a enseñar y aprender trascendiendo los supuestos de uso instrumental.

La repercusión de estos cambios en este ámbito se tradujo en la redefinición de los planes de estudio de carreras de grado de la Facultad de referencia, con la incorporación de asignaturas dentro del área Tecnologías Educativas. A la vez, surgió la demanda de innovaciones didácticas mediadas por entornos virtuales de enseñanza y aprendizaje (EVEA) en el sistema bimodal impulsado por la política universitaria. En este escenario, focalizamos conocimientos, prácticas docentes y construcciones del CDTC en las aulas de las asignaturas del área citada, como modos de manifiestación del CPD.

El inicio de la línea de investigación sobre el Conocimiento Didáctico del Contenido (CDC) se adjudica a Shulman (1986), quien generó en la comunidad especializada uno de los programas de investigación más fecundo. Shulman y seguidores no incorporaron las tecnologías como un elemento destacado del CPD (Marcelo, Yot y Perera, 2016), por lo que la proliferación de investigaciones que resignifican este constructo datan poco más de una década.

Éste, como mencionáramos, se renovó con los aportes de Mishra y Koehler (2006), Koehler, Mishra y Cain (2015), dando lugar al Technological Pedagogical Content Knowledge (TPACK), traducido como Conocimiento Didáctico-Tecnológico del Contenido (CDTC).

El Modelo TPACK (Mishra y Koehler, 2006) incluye tres dimensiones: conocimiento disciplinar (CK, Content Knowledge), conocimiento pedagógico (PK, Pedagogical Knowledge) y conocimiento 
Revista de la Escuela de Ciencias de la Educación, año 15, nRo. 14, vol 2. Julio a Diciembre de 2019. PÁGinas 14-27. ISSN 18516297 (DESDE DICIEMBRE DE 2006 A DICIEMBRE DE 2017). ISSN 2362-3349 (EN LÍNEA). EL MODELO TPACK EN LA PRAXIS DOCENTE EN UNA UNIVERSIDAD ARGENTINA. CONOCIMIENTOS Y PRÁCTICAS DOCENTES EN TORNO AL CONOCIMIENTO DIDÁCTICO-TECNOLÓGICO DEL CONTENIDO (CDTC) en aulas universitarias. Fernando, A. Flores. Margarita, C. Ortiz.

tecnológico (TK, Technological Knowledge). Su importancia radica en el énfasis en las nuevas formas de conocimiento emergentes de la intersección entre cada uno de éstos (Figura 1):

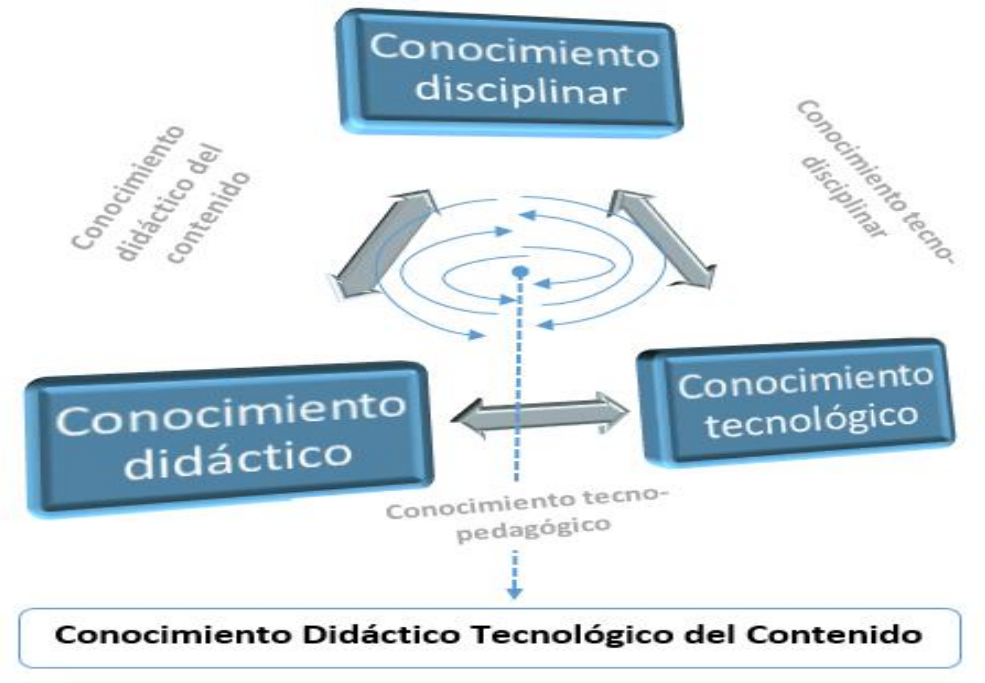

Figura 1: Componentes del modelo TPACK.

Fuente: elaboración propia.

Este modelo desarrollado y extendido en EE.UU amplió sus horizontes en los últimos tiempos tanto en investigación como en su utilización en otros ámbitos. Se demostró su eficacia en variados contextos educativos y niveles de enseñanza y en acciones investigativas y de formación del profesorado; sin obviar la construcción de un modelo teórico que garantice la comprensión del comportamiento de las TIC en los procesos de enseñanza y aprendizaje (Anderson, Barham y Northcote, 2013).

Por su parte, Angeli y Valanides (2009) y Cabero Almenara (2014) complementan y amplían este marco de saberes por considerarlo excesivamente generalista, incluyendo el contexto del proceso; las ideas acerca del aprendizaje; la estructura organizativa; la auto-percepción en el uso de las TIC; los elementos intangibles (cuestiones ideológicas y éticas) que enmarcan al curriculum y la acción del docente en el escenario concreto donde actúa. De este modo, conciben al modelo como un cuerpo de conocimientos que hacen que el profesor sea competente en el diseño de una propuesta de enseñanza y aprendizaje.

Las explicaciones y matices de las dimensiones del modelo, elaborados por Schmidt, Baran, Thompson, Mishra, Koehler y Shín (2009), fueron recogidos y expuestos en la obra de Cabero Almenara (2014), cuya síntesis aparece en la Tabla 1 que, además incluye una tercera columna de indicadores, construidos a partir de la propuesta del TPACK competencial de Cejas-León, Navío-Gámez y BarrosoOsuna (2016).

\begin{tabular}{|c|c|c|}
\hline Categorías & Definiciones & Indicadores \\
\hline $\begin{array}{c}\text { Conoc. del } \\
\text { Contenido } \\
\text { (CC/CK) }\end{array}$ & $\begin{array}{l}\text { Conocimiento del contenido a } \\
\text { enseñar. Representaciones sobre } \\
\text { temas específicos en un área } \\
\text { determinada }\end{array}$ & $\begin{array}{l}\text { Dominio del estado del arte de su disciplina. } \\
\text { Actualización constante en su disciplina. } \\
\text { Reconocimiento de lagunas disciplinares. } \\
\text { Participación activa en redes disciplinares. } \\
\text { Análisis y reflexión crítica sobre } \\
\text { problemáticas dentro de la disciplina. }\end{array}$ \\
\hline $\begin{array}{l}\text { Conoc. } \\
\text { Didáctico } \\
\text { (CD/PK) }\end{array}$ & $\begin{array}{l}\text { Conocimiento de las actividades } \\
\text { pedagógicas generales que podrían } \\
\text { utilizarse, de los procesos y prácticas, } \\
\text { del método de enseñanza y de sus } \\
\text { relaciones con el pensamiento y los } \\
\text { propósitos educativos. }\end{array}$ & $\begin{array}{l}\text { Tutorización del proceso de enseñanza- } \\
\text { aprendizaje para favorecer la autonomía del } \\
\text { alumno. } \\
\text { Utilización de estrategias didácticas } \\
\text { multivariadas. } \\
\text { Evaluación el aprendizaje. } \\
\text { Diseño de la programación didáctica. }\end{array}$ \\
\hline $\begin{array}{l}\text { Conoc. } \\
\text { Tecnológ. } \\
\text { (CT/TK) }\end{array}$ & $\begin{array}{l}\text { Conocimiento sobre el funcionamiento } \\
\text { de las tecnologías y de los modos de } \\
\text { presentación para desarrollar una } \\
\text { actividad profesional. }\end{array}$ & $\begin{array}{l}\text { Resolución de problemas técnicos. } \\
\text { Asimilación de conocimientos tecnológicos. } \\
\text { Actualización sobre las tecnologías } \\
\text { importantes. } \\
\text { Comunicación en entornos digitales. }\end{array}$ \\
\hline
\end{tabular}


Revista de la Escuela de Ciencias de la Educación, año 15, nRo. 14, vol 2. Julio a Diciembre de 2019. PÁGinas 14-27. ISSN 18516297 (DESDE DICIEMBRE DE 2006 A DICIEMBRE DE 2017). ISSN 2362-3349 (EN LÍNEA). EL MODELO TPACK EN LA PRAXIS DOCENTE EN UNA UNIVERSIDAD ARGENTINA. CONOCIMIENTOS Y PRÁCTICAS DOCENTES EN TORNO AL CONOCIMIENTO DIDÁCTICO-TECNOLÓGICO DEL CONTENIDO (CDTC) en aulas universitarias. Fernando, A. Flores. Margarita, C. Ortiz.

\begin{tabular}{|c|c|c|}
\hline & & Protección y seguridad. \\
\hline $\begin{array}{c}\text { Conoc. } \\
\text { Didáctico } \\
\text { del } \\
\text { Contenido } \\
\text { (CDC/PCK) }\end{array}$ & $\begin{array}{l}\text { Es aquel situado en un área concreta } \\
\text { de conocimientos. Se construye } \\
\text { mediante una síntesis idiosincrásica } \\
\text { entre el conocimiento de la materia, el } \\
\text { conocimiento pedagógico general y el } \\
\text { conocimiento de los alumnos y es } \\
\text { afectado por la trayectoria profesional } \\
\text { del docente. }\end{array}$ & $\begin{array}{l}\text { Tutorización en las disciplinas que imparte. } \\
\text { Evaluación del contenido disciplinar. } \\
\text { Programación didáctica de las disciplinas } \\
\text { que imparte. } \\
\text { Estrategias didácticas para guiar el } \\
\text { aprendizaje disciplinar. } \\
\text { Selección de estrategias didácticas acorde } \\
\text { al contenido disciplinar. }\end{array}$ \\
\hline $\begin{array}{l}\text { Conoc. } \\
\text { Tecno } \\
\text { disciplinar } \\
\text { (CCT/TCK) }\end{array}$ & $\begin{array}{l}\text { Refiere al cómo representar conceptos } \\
\text { con la tecnología y cómo con ésta es } \\
\text { posible crear otras, para contenidos } \\
\text { específicos. Es independiente del } \\
\text { conocimiento acerca de su uso en un } \\
\text { contexto pedagógico. }\end{array}$ & $\begin{array}{l}\text { Representación de los contenidos } \\
\text { disciplinares con tecnologías concretas. } \\
\text { Tecnología en la actualización y en redes } \\
\text { disciplinares. }\end{array}$ \\
\hline $\begin{array}{c}\text { Conoc. } \\
\text { Tecno- } \\
\text { pedagógic } \\
0 \\
\text { (CDT/TPK) }\end{array}$ & $\begin{array}{l}\text { Es el conocimiento de las actividades } \\
\text { pedagógicas generales con utilización } \\
\text { de tecnologías en la enseñanza. }\end{array}$ & $\begin{array}{l}\text { Tecnología en el proceso de enseñanza- } \\
\text { aprendizaje favoreciendo la autonomía del } \\
\text { alumno. } \\
\text { Tecnología en la evaluación. } \\
\text { Selección de tecnologías para actividades } \\
\text { didácticas. } \\
\text { Reflexión y pensamiento crítico sobre el uso } \\
\text { de las tecnologías en el proceso de } \\
\text { enseñanza-aprendizaje. } \\
\text { Uso ético de las tecnologías y en la } \\
\text { retroalimentación para mejorar el } \\
\text { aprendizaje de los discentes. }\end{array}$ \\
\hline $\begin{array}{l}\text { Conoc. } \\
\text { Didáctico } \\
- \\
\text { Tecnológ. } \\
\text { del } \\
\text { Contenid } \\
\text { o (CDTC/ } \\
\text { TPACK) }\end{array}$ & $\begin{array}{l}\text { Conocimiento sobre la coordinación } \\
\text { del uso de las actividades específicas } \\
\text { de las materias y contenidos para } \\
\text { facilitar el aprendizaje mediante uso de } \\
\text { las TIC. Alude a la integración de la } \\
\text { tecnología en la enseñanza de un } \\
\text { contenido disciplinar. }\end{array}$ & $\begin{array}{l}\text { Guía y ayuda a terceros a combinar los } \\
\text { contenidos disciplinares, las tecnologías y } \\
\text { estrategias didácticas. } \\
\text { Impartición de lecciones que combinan } \\
\text { tecnología, contenido disciplinar y } \\
\text { estrategias didácticas. } \\
\text { Selección de tecnologías que mejoran los } \\
\text { contenidos, la forma de impartirlos y lo que } \\
\text { aprende el alumnado. }\end{array}$ \\
\hline
\end{tabular}

En este artículo analizamos la configuración y expresión del TPACK en la praxis docente en asignaturas de las Tecnologías educativas y el modo en que intersectan la investigación y la gestión en este constructo. La caracterización que realizan diversos autores sobre el profesorado universitario goza de un marcado consenso respecto de cuáles son las funciones o dimensiones de este colectivo: docencia, investigación, gestión (Gewerc, 2001; Cruz Tomé, 2003; Demuth y Sánchez, 2017).

Tomamos como antecedentes diversos estudios. Por un lado, los usos tecnológicos que demandan capacidades básicas se asocian a prácticas con foco en el docente y las que requieren capacidades más complejas se vinculan a enfoques constructivistas (Ertmer, 2005).

Otros, destacan la influencia de las creencias de los docentes sobre la utilización de las tecnologías en el aula (Ertmer, 2005; Marcelo y Vaillant, 2009; Arancibia, Soto y Contreras, 2010) y señalan que las mismas están muy asentadas en torno al enseñar y aprender con TIC, se relacionan con las decisiones que adoptan y acciones que realizan, previamente, durante y a posteriori de sus prácticas educativas.

Para estos autores, un elemento clave que facilita o dificulta la implicación de un docente en un proyecto de innovación con tecnologías, es la compatibilidad entre éstas y sus creencias pedagógicas. Otras indagaciones, también pusieron al descubierto que los docentes más reflexivos y conscientes de sus propias creencias pedagógicas, generalmente, son más adaptativos y flexibles. Así, la implementación con éxito de tecnologías en el aula es más probable que se produzca cuando los profesores reflexionan acerca de su enseñanza y de los objetivos que persiguen.

Hewe y Brush (2007) en su estudio sobre los obstáculos para el uso innovador de las TIC, identifican barreras directas e indirectas. Las mismas incluyen: a) las actitudes y creencias de los profesores en torno al uso de las TIC; b) sus conocimientos y habilidades; c) la institución (liderazgo, planificación, etc.), y d) recursos. Concluyen que la integración de las TIC es indirectamente influenciada por la cultura institucional, especialmente, en lo relativo a los modelos de enseñanza y evaluación. En 
Revista de la Escuela de Ciencias de la Educación, año 15, nRo. 14, vol 2. Julio a Diciembre de 2019. PÁGinas 14-27. ISSN 18516297 (DESDE DICIEMBRE DE 2006 A DICIEMBRE DE 2017). ISSN 2362-3349 (EN LÍNEA). EL MODELO TPACK EN LA PRAXIS DOCENTE EN UNA UNIVERSIDAD ARGENTINA. CONOCIMIENTOS Y PRÁCTICAS DOCENTES EN TORNO AL CONOCIMIENTO DIDÁCTICO-TECNOLÓGICO DEL CONTENIDO (CDTC) en aulas universitarias. Fernando, A. Flores. Margarita, C. Ortiz.

general, las investigaciones coinciden en que no se contempla la integración de las TIC como un elemento aislado del contexto en que se produce, sino situado y atravesado por éste.

Teniendo en cuenta que la buena enseñanza con TIC se produce en circunstancias particulares y concretas de la práctica de un profesor con un conjunto de alumnos, la enfocamos desde sus conocimientos, experiencias y creencias significativos para la práctica. Desde este lugar, el CDTC se muestra como categoría central del CPD y potente constructo interpretativo y analítico para abordar problemáticas vinculadas con el uso de las TIC en la enseñanza.

\section{Decisiones metodológicas}

Realizamos un estudio de caso colectivo también llamado múltiple. Para Stake (2007) se trata de un sistema integrado en contextos situados, compuesto por casos individuales (cada profesora) unidades de análisis que comparten características comunes estudiadas en un primer momento en su singularidad-, a partir de los cuales planteamos las hipótesis interpretativas. Los trabajos revisados reconocen factores de influjo que abarcan tanto lo social como lo individual (Bolívar, 2005; Gewerc et al., 2013; Cabero Almenara, 2014).

El caso múltiple está constituido por seis casos incrustados: asignaturas de la Licenciatura en Ciencias de la Educación orientación Tecnología Educativa (Educación a Distancia-EaD; Nuevas Tecnologías de la Información y Comunicación-NTIC; Diseño y Producción de Materiales EducativosDPME; Elaboración y Evaluación de Proyecto- EEPE); asignatura de los Profesorados Historia, Letras, Filosofía y Geografía (Tecnología Educativa-TE) y asignatura del Profesorado y Licenciatura en Educación Inicial (Tecnología Educativa en el Nivel Inicial-TENI). La constitución del caso se detalla en la figura 2.

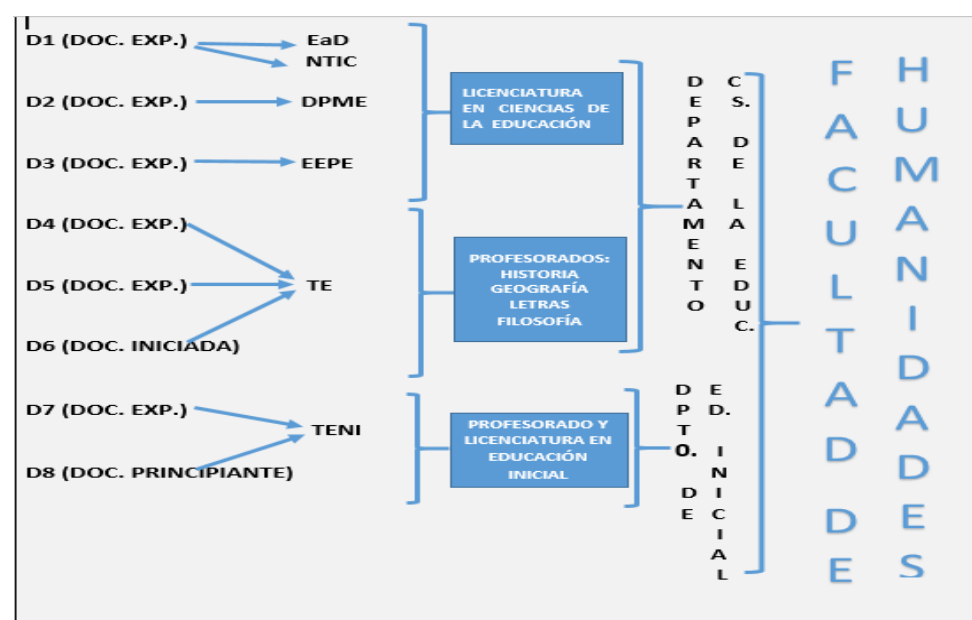

Figura 2: Constitución del caso múltiple. Fuente: elaboración propia.

Para el trabajo de campo empleamos entrevistas semiestructuradas, análisis de documentos profesionales y observaciones no participantes de clases presenciales y virtuales. Concretamente, la secuencia del abordaje metodológico de la investigación siguió los pasos que a continuación detallamos:

- Entrevista Introductoria de presentación de la investigación y las condiciones de participación.

- Entrevista Inicial sobre trayectoria y posición laboral actual en las dimensiones profesional, docencia, investigación y gestión (procesos de formación, aprendizaje profesional docente e interacción con las otras dimensiones).El documento de apoyo: Curriculum Vitae.

* Envío del desgrabado de la entrevista para su revisión y modificación.

- Entrevista de profundización de los componentes del CPD (conocimiento y concepciones disciplinares; pedagógicas; tecnológicas y del contexto). Documento de apoyo: Programas de las asignaturas.

* Envío del desgrabado de la entrevista para su lectura y modificación.

- Ciclo de recogida de un desarrollo temático, en tres etapas: a) entrevista breve acerca de la planificación; b) observación y registro de clases; y c) al finalizar ésta/s, entrevista breve de reflexión para el análisis y evaluación de lo realizado. Documento de apoyo: plan o guión didáctico.

* Envío de los análisis preliminares del ciclo para su lectura y modificación.

- Construcción de los casos particulares.

- Construcción del caso múltiple y comunicación a las protagonistas. 
Revista de la Escuela de Ciencias de la Educación, año 15, nRo. 14, vol 2. Julio a Diciembre de 2019. PÁGinas 14-27. ISSN 18516297 (DESDE DICIEMBRE DE 2006 A DICIEMBRE DE 2017). ISSN 2362-3349 (EN LÍNEA). EL MODELO TPACK EN LA PRAXIS DOCENTE EN UNA UNIVERSIDAD ARGENTINA. CONOCIMIENTOS Y PRÁCTICAS DOCENTES EN TORNO AL CONOCIMIENTO DIDÁCTICO-TECNOLÓGICO DEL CONTENIDO (CDTC) en aulas universitarias. Fernando, A. Flores. Margarita, C. Ortiz.

En todo momento efectuamos instancias de validación -participación de las docentes- de los discursos propios e interpretaciones, con el objetivo de lograr una construcción en la que las protagonistas se reconozcan, sean conscientes de sus posiciones y de la del investigador; y se interroguen por ambas, en coherencia con un estudio de carácter cualitativo, que se inscribe en el Paradigma Hermenéutico (Sandín Esteban, 2003).

\section{Construcción del caso colectivo incrustado}

El caso múltiple queda construido mediante un relato o narrativa que sintetiza los aspectos relevantes (conjunto de equipos docentes), con sus casos incrustados (cada equipo), con la finalidad de encontrar puntos de aproximación y diferencias más conspícuas en este estudio. En las cátedras unipersonales, cada profesora constituye el caso. En la figura 3 se observan los diferentes diseños de estudio de caso, ubicamos nuestro estudio en la última opción.



Figura 3: Tipos de casos.

Fuente: Yin (1994, p. 46).

De este modo, la narrativa se convierte en el proceso recursivo que vuelve a trabajar las ideas más significativas permitiendo una mayor profundización en la comprensión.

Indagar sobre la construcción y manifestación del conocimiento profesional del profesor desde cuatro funciones propias del contexto universitario (profesional, investigación, gestión y docente) que lo exceden al tiempo que lo conforman; implicó establecer una red para aprehenderlo. Asumimos el CDTC como un nudo sustantivo del CPD universitario, esto es, como compleja trama de relaciones que enlaza las funciones identitarias.

Sobre la base de las entrevistas y lectura de los Currículum vitae, caracterizamos a las profesoras participantes del estudio, identificadas con una sigla y número. A excepción de $D 5$, todas son profesoras en Ciencias de la Educación.

\section{Conocimientos y prácticas docentes en torno al CDTC}

Respecto de la incidencia de las funciones del docente universitario en el CDTC, los hallazgos denotan que la dimensión profesional del CPD de este colectivo coincide con las de docencia y extensión "en" y "con" TIC (D1,D2 y D7). Estas experiencias conjuntamente con las agenciadas en las funciones de gestión e investigación, median los modos de configurar el CDTC en D1 y D2 y sólo la última en $D 4$ y $D 7$. En las dos primeras, los conocimientos y experiencias en gestión se traducen en las clases:

La coordinación de los programas con TIC de la Universidad y del Ministerio retroalimentaron mi propuesta pedagógica que tiene una fuerte impronta de las tecnologías educativas en EaD, a la vez que son un objeto de mis proyectos de investigación... estos cargos son dinamizadores y potenciadores de las asignaturas que dicto. (Entrevista D1).

D2, rescata el valor de la experiencia en UNNE-Virtual para la organización de la asignatura:

...fue donde comencé a acercarme a esta área de conocimiento que es el Diseño y Producción de Materiales... comencé a capacitar en eso... en mi cátedra lo enfoco desde esa mirada, pensando sobre todo que ellos tenían la materia NTIC...fui profundizando lo que es el análisis didáctico, del sujeto que aprende, de lo epistemológico... (Entrevista D2). 
Revista de la Escuela de Ciencias de la Educación, año 15, nRo. 14, vol 2. Julio a Diciembre de 2019. PÁGinas 14-27. ISSN 18516297 (DESDE DICIEMBRE DE 2006 A DICIEMBRE DE 2017). ISSN 2362-3349 (EN LÍNEA). EL MODELO TPACK EN LA PRAXIS DOCENTE EN UNA UNIVERSIDAD ARGENTINA. CONOCIMIENTOS Y PRÁCTICAS DOCENTES EN TORNO AL CONOCIMIENTO DIDÁCTICO-TECNOLÓGICO DEL CONTENIDO (CDTC) en aulas universitarias. Fernando, A. Flores. Margarita, C. Ortiz.

\section{EI CDTC en la praxis docente}

En la dimensión docente, analizamos la categoría y subcategorías emergentes del CDTC de las docentes, a la luz del modelo TPACK:

\begin{tabular}{|c|c|c|}
\hline Categoría & Sub-categorías & Definición \\
\hline \multirow{7}{*}{$\begin{array}{l}\text { CDTC (Conocimiento Didáctico } \\
\text { Tecnológico del Contenido) }\end{array}$} & Status del CDTC & $\begin{array}{l}\text { Lugar del CDTC en el } \\
\text { conocimiento profesional docente. }\end{array}$ \\
\hline & Autopercepción uso de TIC & $\begin{array}{l}\text { Percepción propia sobre el uso de } \\
\text { las TIC en la asignatura. }\end{array}$ \\
\hline & $\begin{array}{l}\text { Tipo y frecuencia de uso de } \\
\text { las TIC }\end{array}$ & $\begin{array}{l}\text { Modalidades y periodicidad del uso } \\
\text { de las TIC en la enseñanza. }\end{array}$ \\
\hline & $\begin{array}{c}\text { Visiones sobre la } \\
\text { integración del CDTC en a } \\
\text { praxis }\end{array}$ & $\begin{array}{l}\text { Imágenes acerca de las formas de } \\
\text { integración del CDTC en la } \\
\text { práctica docente. }\end{array}$ \\
\hline & $\begin{array}{c}\text { Características de } \\
\text { enseñanza y aprendizaje } \\
\text { con tecnologías }\end{array}$ & $\begin{array}{l}\text { Caracterización de las propuestas } \\
\text { concretas de enseñanza con TIC. }\end{array}$ \\
\hline & $\begin{array}{l}\text { Manifestaciones del CDTC } \\
\text { en un ciclo didáctico }\end{array}$ & $\begin{array}{l}\text { Descripción acerca de los modos } \\
\text { de expresión del CDTC en una } \\
\text { secuencia didáctica de la } \\
\text { asignatura. }\end{array}$ \\
\hline & $\begin{array}{c}\text { Facilitadores y limitantes en } \\
\text { torno a la integración de las } \\
\text { TIC }\end{array}$ & $\begin{array}{l}\text { Identificación de factores } \\
\text { posibilitadores y obstaculizadores } \\
\text { a nivel personal e institucional para } \\
\text { la integración de las TIC. }\end{array}$ \\
\hline
\end{tabular}

En este apartado, presentamos los casos de las diferentes carreras y luego, describimos narrativamente el caso múltiple incrustado. Cabe señalar que por razones de espacio, profundizamos aquellas subcategorías que nos permiten mostrar la coherencia e inconsistencias entre lo que dicen y piensan (autopercepciones y visiones) y lo que hacen (propuestas y manifestaciones en el ciclo didáctico) las profesoras protagonistas.

\section{- Licenciatura en Ciencias de la Educación}

En la siguiente Tabla caracterizamos el perfil profesional de las docentes de esta carrera.

\begin{tabular}{l|ll}
\hline Doc & Asignatura & Características \\
\hline D1 & EaD-NTIC & $\begin{array}{l}\text { Profesora experimentada e investigadora categorizada. Adjunta con } \\
\text { dedicación simple desde } 2005 \text { y } 2015 \text { respectivamente. Acredita formación } \\
\text { de posgrado en Tecnologías Educativas y experiencia en la dirección del } \\
\text { Programa UNNE-Virtual y Conectar Igualdad (gestión). Actualmente, } \\
\text { Doctora en Antropolgía Social, coordina y dicta cursos de grado y posgrado } \\
\text { acerca de las TIC. Directora de Proy. de Investigación en Facultad de } \\
\text { Odontología. }\end{array}$ \\
\hline $\mathbf{D 2}$ & DPME & $\begin{array}{l}\text { Docente experimentada. Adjunta con dedicación simple desde 2005. En el } \\
\text { Programa UNNE-virtual tuvo a su cargo la coordinación del área Diseño de } \\
\text { materiales (gestión). Dictante y tutora de cursos de grado y posgrado en } \\
\text { Educación virtual en la Universidad y otros niveles educativos. } \\
\text { Docente experimentada e investigadora categorizada (Didáctica). Adjunta } \\
\text { con dedicación simple desde 2005. Magister en Tecnología educativa y } \\
\text { Diplomada en Educación y Nuevas Tecnologías. }\end{array}$ \\
\hline
\end{tabular}

Tabla 3: Asignaturas unipersonales.

Fuente: elaboración propia.

D1 considera que existe una directa relación entre su autopercepción en el uso de las TIC y los modos de integrarlas en sus asignaturas:

Su manejo no garantiza que seas un buen docente, es importante conocer lo que hay y para qué se usa, afirma que lo distintivo de un buen docente es la planificación de cómo enseñar determinados contenidos para $x$ grupo y cómo juegan las TIC en ella... qué rol 
Revista de la Escuela de Ciencias de la Educación, año 15, nRo. 14, vol 2. Julio a Diciembre de 2019. PÁGinas 14-27. ISSN 18516297 (DESDE DICIEMBRE DE 2006 A DICIEMBRE DE 2017). ISSN 2362-3349 (EN LÍNEA). EL MODELO TPACK EN LA PRAXIS DOCENTE EN UNA UNIVERSIDAD ARGENTINA. CONOCIMIENTOS Y PRÁCTICAS DOCENTES EN TORNO AL CONOCIMIENTO DIDÁCTICO-TECNOLÓGICO DEL CONTENIDO (CDTC) en aulas universitarias. Fernando, A. Flores. Margarita, C. Ortiz.

ocupan, por ejemplo, en el aula virtual, pretender que el alumno la utilice, participe, que sirva como un espacio de enseñanza y aprendizaje, implica modificar lo presencial. La utilización de todas las funciones, lo hace un docente experto en el manejo de la plataforma, pero si su planificación es deficiente, difícilmente producirá una buena experiencia....Me fascina el desafío...ver si lo que pongo en práctica...es coherente con lo que está pasando, tengo que ser abierta en ese sentido...tengo dos asignaturas que están en un constante cambio (Entrevista D1).

Por su parte D2, focaliza la mirada en los aprendizajes que pretende que "logren los alumnos cuando se vinculan al uso de la TIC en -el diseño de- un material didáctico" (Entrevista D2), mientras que D3 percibe su materia (EEPE) como tecnología educativa y que "la inserción de las nuevas herramientas está al servicio de, pero lo que sostiene todo sigue siendo tecnología educativa" (Entrevista D3). En cuanto a las visiones de integración de las tic en la praxis docente, D1 asume que "las tecnologías no son las que inducen cambios, sino las acciones, los procesos que se generan con ellas, los que permiten la producción de ciertos saberes y conocimientos, a partir de los cuales se pueden transformar las prácticas" (Entrevista D1). Entiende que asociar los cambios en la enseñanza con la mera aplicación de las TIC, supone hacerlo de modo superfluo.

Para D2, las TIC son herramientas que -conjuntamente- con elementos contextuales y didácticos pueden ayudar a mejorar los procesos de enseñanza:

...Yo no les enseño a usar recursos tecnológicos... no me ato a la tecnología...que no piensen que tienen que saber los softwares...sino, qué quiero y cómo lo pienso. Ahora, vamos a armar un video educativo, les doy las pautas... cuáles son los contenidos mínimos, cómo lo arman, qué objetivos, para qué... (Entrevista D2).

D3, sustenta una visión acerca de su incorporación en los proyectos educativos basada en la reflexión crítica del peso de lo didáctico en lo tecnológico.

Respecto de las características de las propuestas didácticas con TIC, éstas denotan coherencia teoría-práctica en EaD y en NTIC arman un proyecto posicionado desde diferentes roles del perfil profesional:

En consistencia con el grupo de aprendizaje y la especificidad de la asignatura, concibo el aprendizaje vivencial, particularmente de la $\mathrm{EaD}$, integrando teoría y práctica; vivencias que permiten identificar la necesidad de contar con materiales claros y completos, recibir apoyo tutorial permanente, organizar el tiempo de estudio, manejar el EVA, entre otros. (Entrevista D1).

D2 manifiesta que "los estudiantes deben tener en claro que la tecnología no es el centro, sus propuestas con inclusión de TIC tienen que partir de una necesidad" y para diseñar materiales, también los posiciona en "variados roles y funciones en equipos interdisciplinarios", trabajando de modo articulado con D3, "En (EEPE), habían armado un proyecto de capacitación para Amas de Casa (Educación no formal), y trabajamos los componentes inherentes a la producción de materiales educativos mediados por las TIC" (Entrevista D2).

Por su lado, D3, compara su asignatura con otras de la orientación y aclara "trabajo la herramienta (proyecto) como dispositivo tecnológico, cómo elaborar un proyecto de EaD o para producir materiales educativos" (Entrevista D3).

Estos relatos se sustancian en la subcategoría referida a las manifestaciones del CDTC en un ciclo didáctico, con construcciones metodológicas y configuraciones didácticas que dan cuenta de la dialéctica entre lo universal (CDTC), lo singular (cada profesora, equipo) y lo particular (caso múltiple incrustado).

D1, expresa que:

...en EaD no es necesario verlos para que ellos puedan aprender... los hago atravesar por una experiencia real y concreta de ser un estudiante a distancia, los pongo en un rol real... como observaste en esta unidad, en la que el trabajo la enseñanza y aprendizaje es con una propuesta concreta, una situación lo más cercana posible a la experiencia, o sea, posicionándose en el rol de tutores de distancia o la de analizar los componentes de las propuestas de EaD de otras universidades. En NTIC, no sólo les digo que usar una plataforma virtual para hacer lo mismo que en la enseñanza presencial, no es una buena experiencia con tecnología, sino que cada recurso implica otras prácticas, una buena experiencia debe contemplarla de un modo global, tal como ves en la plataforma virtual. (Reg. Obs. Clase, D1) 
Revista de la Escuela de Ciencias de la Educación, año 15, nRo. 14, vol 2. Julio a Diciembre de 2019. PÁGinas 14-27. ISSN 18516297 (DESDE DICIEMBRE DE 2006 A DICIEMBRE DE 2017). ISSN 2362-3349 (EN LÍNEA). EL MODELO TPACK EN LA PRAXIS DOCENTE EN UNA UNIVERSIDAD ARGENTINA. CONOCIMIENTOS Y PRÁCTICAS DOCENTES EN TORNO AL CONOCIMIENTO DIDÁCTICO-TECNOLÓGICO DEL CONTENIDO (CDTC) en aulas universitarias. Fernando, A. Flores. Margarita, C. Ortiz.
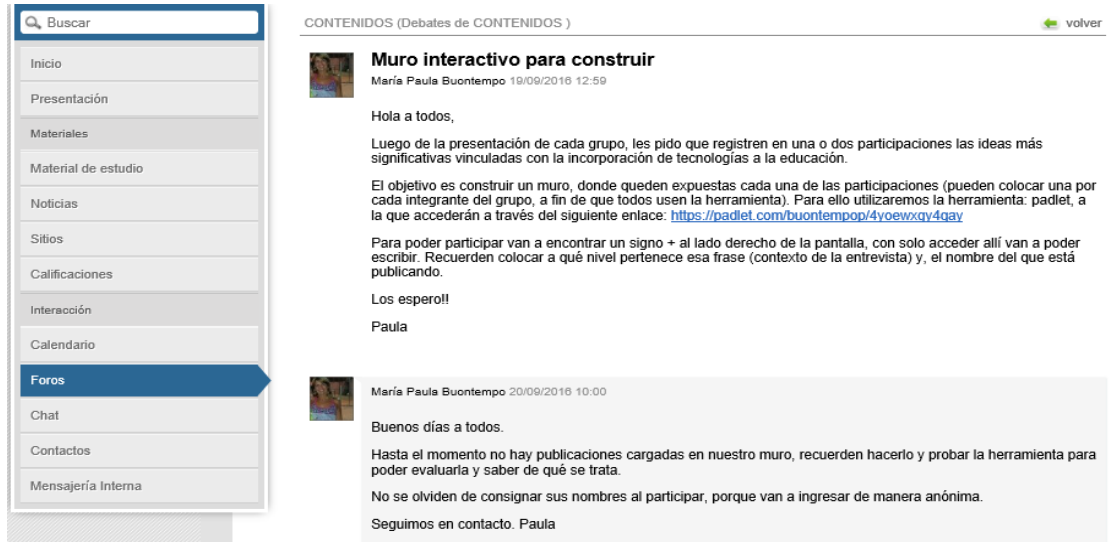

Figura 4: Captura de pantalla del Aula Virtual de NTIC Fuente: elaboración propia.

D2, expresa:

Pretendo que armen, produzcan y experimenten el proceso del diseño de materiales conjugando distintos componentes...adopté y adecué las actividades de un curso virtual. Les pareció novedoso a los alumnos...como vieron que en este momento están diseñando materiales digitalizados para una capacitación para docentes de secundaria con tecnologías digitales" (Reg. Obs. Clase, D2).

En EEPE, observamos que la docente manifestó al inicio de las clases que "seleccionarán un proyecto tecnológico educativo, lo analizarán y sugerirán propuestas de mejoramiento...Por último, producirán un proyecto de innovación tecnológica" (Reg. Obs. Clase, D3).

\section{- Profesorados en Historia, Geografía, Letras y Filosofía}

\begin{tabular}{l|l|ll}
\hline & Titular & Auxiliares Docentes \\
\hline D4 & $\begin{array}{l}\text { Profesora experimentada e investigadora } \\
\text { categorizada. De 2003 a 2015, adjunta y luego, }\end{array}$ \\
titular con dedicación exclusiva. Especialista y & \\
Magister en Docencia Universitaria. Ocupó \\
cargos de gestión en la unidad académica y \\
$\begin{array}{l}\text { Rectorado (no vinculados con las TIC). } \\
\text { Directora de Proy de Investigación sobre } \\
\text { Tecnología Educativa }\end{array}$ \\
\hline D5
\end{tabular}

D4, considera que existe una clara relación entre su autopercepción de las tecnologías y los modos de integrarlas. Busca transformar o transparentar estas percepciones en fundamentos y propósitos: "...las TIC son herramientas, no son un gran hermano que maneja al docente al ritmo de lo novedoso y las modas" (Entrevista D4). Considera que a pesar de no estar en la vanguardia de los avances tecnológicos no deja de ser especialista en Tecnología educativa y que la enseñanza de esta disciplina requiere de un equipo interdisciplinario.

D5, evalúa su práctica con TIC de modo positivo, "en este momento lo que está en auge son las herramientas en la nube que son justamente lo que tratan de utilizar...antes de emplearlas, experimentamos en el equipo su funcionalidad y adecuación con los contenidos" (Entrevista D5).

En cambio, D6 sostiene que existe cierta relación entre su autopercepción del uso de las TIC e integración de éstas. Reconoce que algunas veces no es coherente ".. pretendo usar nuevos recursos en la clase y termino empleando la tiza y el pizarrón...uso tecnologías digitales pero después en la clase 
Revista de la Escuela de Ciencias de la Educación, año 15, nRo. 14, vol 2. Julio a Diciembre de 2019. PÁGinas 14-27. ISSN 18516297 (DESDE DICIEMBRE DE 2006 A DICIEMBRE DE 2017). ISSN 2362-3349 (EN LÍNEA). EL MODELO TPACK EN LA PRAXIS DOCENTE EN UNA UNIVERSIDAD ARGENTINA. CONOCIMIENTOS Y PRÁCTICAS DOCENTES EN TORNO AL CONOCIMIENTO DIDÁCTICO-TECNOLÓGICO DEL CONTENIDO (CDTC) en aulas universitarias. Fernando, A. Flores. Margarita, C. Ortiz.

no logro implementarlas...no puedo resolver esa diferencia como lo haría un experto al integrarlas" (Entrevista D6).

Con respecto a las visiones de integración de las TIC en la praxis docente, indica D5 que, en las propuestas de enseñanza y aprendizaje, éstas vienen por separado "...están aparte como algo que se anexa" (Entrevista D5). Estima que el mejor criterio es que estén integradas en la secuencia didáctica. Por su parte, D4 señala:

Se está restringiendo la mirada a las tecnologías informáticas y no es solamente eso... en educación se hace tecnologías blandas cuando se habla de dispositivos... en mi materia trato de generar condiciones para la reflexión y el análisis crítico de su inclusión (Entrevista D4).

De igual modo, D6, se muestra crítica ante "aquellos que exaltan rápidamente a la tecnología. El docente tiene que dictar clases y no estar pendiente a la tecnología... no son neutras, tienen que ser facilitadoras" (Entrevista D6).

En torno a las características de las propuestas didácticas con TIC, D4 señala que:

...todos los años se realizan graduales modificaciones al programa ... una vuelta de tuerca, cambio algún autor, actualizo enfoques, trato de no caer en una cuestión meramente instrumental aunque no descarto trabajar con alguna puntualmente, siempre que su uso esté claramente fundamentado (Entrevista D4).

\section{En términos de D5, la propuesta es:}

Una continuación y articulación con lo dado en Didáctica y en otras áreas afines. La secuencia de actividades es más bien proactiva, se facilita recursos, para que ellos trabajen y luego hagan una puesta en común, finalmente se realiza una evaluación de la actividad (Entrevista D5).

Desde la perspectiva de D6, una buena propuesta presenta una sólida fundamentación pedagógico didáctica, contextualizada y las TIC se integran a ese entramado. En sus clases "cae sin querer en lo tradicional... porque el alumno está esperando recibir información” (Entrevista D6).

En relación con las manifestaciones del CDTC en un ciclo didáctico, observamos que en las clases, D4 desarrolla la clase con el contenido del aula virtual, previa solicitud a los alumnos de bajar el material para el trabajo en grupo. Además, proyecta videos al inicio o al cierre de las instancias presenciales (Reg Obs.Clase,D4).

Por su parte, D5 en las clases utiliza presentaciones en Power Point y, ocasionalmente, Prezzi. Trabaja con conferencias cortas de una especialista española en Tecnología educativa. Otros aplicativos para la instancia no presencial son: Google Drive, Grupo Yahoo y Blog. Las inicia con actividades de recuperación de conocimientos previos, a modo de hilo conductor entre lo dado y lo nuevo.

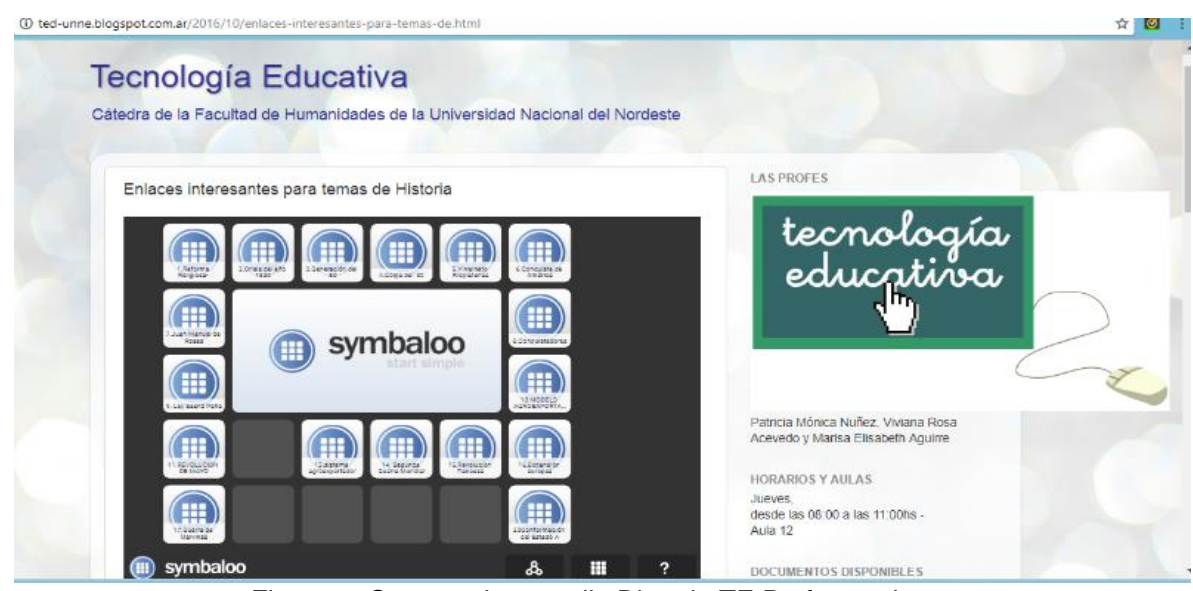

Figura 5: Captura de pantalla Blog de TE Profesorados.

Fuente: elaboración propia.

Aunque D6 reconoce la importancia de las tecnologías digitales en la enseñanza, al inicio de sus clases utiliza Power Point para la exposición de conceptos, como organizador de ideas y luego propone actividades de elaboración grupal, con tareas diferenciadas según las carreras (Reg. Obs. Clase, D6). 
Revista de la Escuela de Ciencias de la Educación, año 15, nRo. 14, vol 2. Julio a Diciembre de 2019. PÁGinas 14-27. ISSN 18516297 (DESDE DICIEMBRE DE 2006 A DICIEMBRE DE 2017). ISSN 2362-3349 (EN LÍNEA). EL MODELO TPACK EN LA PRAXIS DOCENTE EN UNA UNIVERSIDAD ARGENTINA. CONOCIMIENTOS Y PRÁCTICAS DOCENTES EN TORNO AL CONOCIMIENTO DIDÁCTICO-TECNOLÓGICO DEL CONTENIDO (CDTC) en aulas universitarias. Fernando, A. Flores. Margarita, C. Ortiz.

\section{- Profesorado y Licenciatura en Educación Inicial}

\begin{tabular}{|c|c|c|}
\hline & Adjunta & Auxiliar Docente \\
\hline D7 & $\begin{array}{l}\text { Docente experimentada con dedicación simple desde } \\
\text { 2008. Investigadora categorizada. Actualmente, tesista } \\
\text { de la Maestría Procesos educativos mediados por TIC e } \\
\text { integrante del equipo técnico pedagógico del campus } \\
\text { virtual de la Facultad de Medicina de la UNNE. Dirige } \\
\text { proyectos de Investigación en Facultad de Medicina y } \\
\text { dicta cursos de posgrado sobre virtualidad }\end{array}$ & \\
\hline D8 & & $\begin{array}{l}\text { Ingresó en } 2012 \text { con dedicación } \\
\text { simple. Profesora principiante. } \\
\text { Actualmente cursa posgrado } \\
\text { relacionado con Tecnología } \\
\text { educativa. }\end{array}$ \\
\hline
\end{tabular}

Tabla 5: Equipo docente de TENI.

Fuente: elaboración propia.

La autopercepción del uso e integración de las TIC de D7 refiere a los usos instrumental y constructivo de las tecnologías "La perspectiva instrumental coloca el acento en enseñar a utilizar las aplicaciones. En la asignatura no se apunta eso" (Entrevista D7).

D8 manifiesta ser "hábil para el uso de las tecnologías y además como soy del nivel mi relación con las estudiantes es más cercana y les llego al usarlas" (Entrevista D8).

Los relatos acerca de las visiones de integración de las TIC en la praxis docente, en D7 anclan en el propósito de "acercar las TIC, analizar su incidencia en los aprendizajes, pensar cómo atraviesan a las personas...necesitan comprender las lógicas digitales, definir su peso en la asignatura, construir saberes y competencias necesarios para el desenvolvimiento profesional en el mundo actual" (Entrevista D7).

Su par, D8, comenta que:

...en su formación de posgrado fue cuando sistemáticamente comenzó a trabajar con las NTIC. Mi contribución en la asignatura se orienta a la apropiación de las TIC por parte de las estudiantes, porque advierto que son formas distintas de producir y relacionarse con los conocimientos (Entrevista D8).

Las propuestas didácticas con TIC, se caracterizan según D7 en dos encuentros presenciales con la modalidad de aula taller (dos módulos) y trabajo " en el aula de informática con el uso de alguna aplicación, las formas de descargarlas, realizar determinadas tareas como uso efectivo de buscadores..." (Entrevista D7).

D8, destaca que actualmente

...la actitud de las alumnas frente a las tecnologías digitales es más bien receptiva, tanto con el aula virtual como con el correo electrónico. Dado que tienen mayor acceso a los dispositivos móviles, trabajamos con organizadores gráficos digitales, arman mapas conceptuales con el Cmaptool... (Entrevista D8).

Hallamos que las manifestaciones del CDTC en un ciclo didáctico en las clases de D7 se basan en el análisis de casos, lectura de artículos y resolución de problemas, con la finalidad de que puedan "entender qué ocurre al aplicarlas en las clases, qué se puede hacer, cómo se pude construir colaborativamente los conocimientos, de qué modo se puede interactuar, cuáles son los peligros que existen, etc." (Reg.Obs. Clase, D7).

En los Trabajos prácticos en la Sala de Informática, realizan la búsqueda y análisis de páginas web destinadas a docentes de Educación Inicial "desde una perspectiva pedagógica y tecnológica para que ellas puedan ver el panorama docente, además se pretende puedan hacer contacto con profesores de esta área y de otros contextos" (Reg. Obs. Clases, D8).

\section{Unidad y diversidad en el caso múltiple incrustado}

De acuerdo con el análisis de las subcategorías del constructo CDTC en las aulas universitarias, podemos inferir tendencias respecto de algunas de las categorías del Modelo TPACK en los múltiples casos (asignaturas y equipos docentes) con diseño incrustado construido.

La tendencia hacia el Conocimiento Didáctico Tecnológico (CDT) emerge en los casos de asignaturas unipersonales ( 2 y 3 ) y en los dos equipos docentes: 4-6 y 7-8, respectivamente. En éstos la 
Revista de la Escuela de Ciencias de la Educación, año 15, nRo. 14, vol 2. Julio a Diciembre de 2019. Páginas 14-27. ISSN 18516297 (DESDE DICIEMBRE DE 2006 A DICIEMBRE DE 2017). ISSN 2362-3349 (EN LÍNEA). EL MODELO TPACK EN LA PRAXIS DOCENTE EN UNA UNIVERSIDAD ARGENTINA. CONOCIMIENTOS Y PRÁCTICAS DOCENTES EN TORNO AL CONOCIMIENTO DIDÁCTICO-TECNOLÓGICO DEL CONTENIDO (CDTC) en aulas universitarias. Fernando, A. Flores. Margarita, C. Ortiz.

Tecnología es un componente del proceso de enseñanza y aprendizaje para favorecer la autonomía del alumno y en general, son seleccionadas al planificar, implementar y evaluar las actividades didácticas como para la retroalimentación de los aprendizajes reconociendo su potencial constructivo de conocimientos, saberes y competencias.

La reflexión y el pensamiento crítico acerca del uso de las tecnologías en el proceso de enseñanza y aprendizaje aparece explícitamente en D3, D4 y D7, lo que podría vincularse con la formación continua en el área, la formación de grado, el rol y funciones en la asignatura o equipo docente y con el objeto de estudio de la disciplina a cargo.

En casi todos los casos, observamos consistencia entre lo que piensan, dicen y hacen las profesoras, denotando singulares procesos de apropiación de las TIC. Una excepción es D6, cuyo saber práctico y fundamentación teórica no logra cristalizarse en actividades que las integren, dando cuenta de la incoherencia entre discurso y práctica.

En estas prácticas encontramos rasgos que permiten señalar que las TIC aparecen de forma tangencial (Koehler et al. 2015), al margen de los aprendizajes y no de modo integrado. De acuerdo con los niveles de integración curricular de las TIC, los casos están en la interfase entre el uso de las TIC (uso periférico en el aprendizaje y cognición) y la integración curricular de las TIC, es decir, centradas en el aprender (Sánchez, 2003).

Uno de los casos-asignatura unipersonal- muestra tendencia hacia el Conocimiento Didáctico Tecnológico del Contenido (CDTC). La docente (D1) articula contenidos disciplinares, tecnologías y estrategias didácticas y selecciona las tecnologías que coadyuvan a mejorar los contenidos, la forma de abordarlos y los aprendizajes. Las TIC forman la tríada didáctica, bajo el supuesto de generar procesos de producción saberes y conocimientos para transformar las prácticas docentes. Esta transformación se sustenta en los usos de las herramientas digitales y sus implicancias en las actividades que llevan adelante profesores y estudiantes (Coll y Monereo, 2008), y en las que la docente no solo prioriza la adquisición de conocimientos sino su apropiación (Marcelo et al., 2015). En términos de Sánchez (2003) hay incorporación y articulación pedagógica de las TIC en el aula y su apropiación se traduce en su uso invisible y situado y un aprender visible. Aquí, el entorno virtual constituyó un potenciador de la propuesta pedagógico didáctica en lugar de banalizarla (Litwin, 2005), pues los contextos de uso de las TIC generaron dinámicas de innovación y mejora en los procesos de enseñanza y aprendizaje (Coll, 2011).

Finalmente, el Conocimiento Tecnológico (CT) atraviesa el discurso y práctica docente de D5, cuya visibilización de las herramientas digitales se corresponde con un nivel de integración de enfoque más tecnocéntrico del uso de la tecnología para apoyar el aprender (Sánchez, 2003). En la práctica son $D 1, D 2, D 5$ y $D 8$ las que manifestaron ser más proclives a experimentar con las TIC, a pesar de las expresiones relacionadas con la conveniencia de encontrar formas de conjugar estrategias, las TIC y el contenido disciplinar (Roig-Vila, 2016). Coincidimos con Arancibia et al. (2010) en que los constructos implícitos orientan acciones y anclan en las experiencias vividas del sujeto, atravesadas por los procesos formativos y los paradigmas hegemónicos de las prácticas profesionales.

Además, hallamos cierta renuencia al determinismo tecnológico al decir de Lion (2006), postura que las sobrevalora como herramientas indispensables para la innovación y el mejoramiento de las prácticas de la enseñanza, sin considerar las implicancias del proyecto sociocultural, político y pedagógico en el cual se incorporan.

Predomina en el currículo en acción el uso de las TIC de modo subsidiario, con un nivel de integración curricular superficial, a modo de componente lábil (Sánchez, 2003; Kolher et al., 2015). Este tipo de uso dominante da cuenta de una cultura profesional con una naciente internalización del modelo TPACK en la unidad académica. Las epistemologías prácticas diferentes reflejan la existencia de una "tecnología silenciada" en su contenido por el énfasis puesto en las actividades o estrategias, posición que atraviesa la constitución del caso múltiple (Litwin, 2005). En relación con esto, las vinculaciones entre tecnología y conocimiento no son unívocas ni lineales, requiere pensar en la especificidad del soporte, del campo de saber, de la propuesta pedagógica y de los procesos cognitivos que se suscitan con las tecnologías, en el marco de instituciones y clases concretas que los contextualizan (Lion, 2006).

En relación con la dimensión tecnológica en las asignaturas del área en estudio - en el marco del modelo TPACK-, y específicamente, en torno a la especificidad de su enseñanza, advertimos tensiones en las prácticas áulicas entre el conocimiento disciplinar, el conocimiento pedagógico-didàctico y el conocimiento tecnológico, más que en el nivel teórico-discursivo, aunque con matices en las configuraciones analizadas.

Así, lo ontológico (tecnología como objeto de estudio de las disciplinas), lo epistemológico (modos de construcción del conocimiento sobre dicho objeto) y lo metológico (construcciones metodológicas para la inclusiòn curricular de las TIC), por ejemplo imbrincan en la construcción del CDTC en D1 y configuraciones singulares en EaD y NTIC. Dimensiones que en el resto de los casos aparecen desdibujadas o con peso relativo de algunos indicadores de las dimensiones del modelo TPACK. 
Revista de la Escuela de Ciencias de la Educación, año 15, nRo. 14, vol 2. Julio a Diciembre de 2019. Páginas 14-27. ISSN 18516297 (DESDE DICIEMBRE DE 2006 A DICIEMBRE DE 2017). ISSN 2362-3349 (EN LÍNEA). EL MODELO TPACK EN LA PRAXIS DOCENTE EN UNA UNIVERSIDAD ARGENTINA. CONOCIMIENTOS Y PRÁCTICAS DOCENTES EN TORNO AL CONOCIMIENTO DIDÁCTICO-TECNOLÓGICO DEL CONTENIDO (CDTC) en aulas universitarias. Fernando, A. Flores. Margarita, C. Ortiz.

\section{Conclusiones}

A las experiencias en gestión y/o investigación en torno a las TIC, se integran trayectos de formación específica de posgrado en la mayoría de las profesoras, dando lugar a tres categorìas configurativas.

La primera, representada por D1 (EaD y NTIC), caracterizada por la integración curricular de los componentes del modelo TAPCK, enriquecida por la mixtura de las citadas experiencias y trayectoria profesional y en las que las dimensiones sustantiva y sintáctica del conocimiento tecnológicoobjeto de estudio de las Tecnologías educativas- emerge en sucesivas construcciones personales y colectivas en contextos académicos y disciplinas del área. La integración curricular de las TIC evidencia articulación de las tres dimensiones del TPACK en el curriculo en acción: uso situado y centrado en los estudiantes. Integran los procesos de transmisión y construcción del conocimiento.

En el otro polo, la configuraciòn centrada en el conocimiento tecnológico en consistencia con la trayectoria formativa en TIC (D5) amalgama con las construidas por D4 y D6 (CDT) posibilitando el encuentro teoría-práctica en la propuesta didáctico-pedagógica de TE, en estrecha vinculación con el posicionamiento epistemológico en investigación. Prevalece una función periférica de las TIC en el aprendizaje y la cognición, esto es, como recurso de apoyo.

Visibilizamos configuraciones intermedias en las asignaturas unipersonales (DPME y EEPE) y equipo docente de TENI, definidas por la tendencia de rasgos del Conocimiento Didáctico Tecnológico, atravesada por disimiles experiencias formativas y de investigación en la construcción del CDTC, insuficientes para la efectiva articulaciòn de los elementos de éste en sus praxis docentes. La integración de éstas en las prácticas oscila entre el protagonismo en la enseñanza y ser subsidiarias. la tecnología está silenciada en su contenido

Dado que la pauta que conecta a nivel departamental y Unidad Académica es el énfasis en la dimensiòn didáctico-tecnológica del CDCT- posición que atraviesa la constitución del caso multiple,hipotetizamos la existencia de una incipiente cultura de integración de las TIC en la formación docente de grado. Esto implica que el colectivo profesional construya estrategias que actúen como puentes entre tecnologías, vida académica y prácticas profesionales docentes, en el contexto de la bi-modalidad de esta universidad.

\section{Referencias bibliográficas}

- Anderson, A.; Barham, N. \& Northcote, M. (2013). Using the TPACK framework to unite disciplines in online learning. Australasian Journal of Educational Technology, 29(4), pp. 548-565.

- Angeli, C. y Valanides, N. (2009). Epistemological and methodological issues for the conceptualization, development, and assessment of ICT-TPCK: Advances in technological pedagogical content knowledge (TPCK). Computers \& Education, 52(1), pp. 154-168.

- Arancibia, M.; Soto, C. y Contreras, P. (2010). Concepciones del profesor sobre el uso educativo de las Tecnologías de la Información y la Comunicación (TIC) asociadas a procesos de enseñanza-aprendizaje en el aula escolar. Estudios Pedagógicos, 36(1), pp. 23-51.

- Bolívar, A. (2005). Conocimiento didáctico del contenido y didácticas específicas. En Revista de currículum y formación del profesorado, 9, pp. 1-39.

- Cabero Almenara, J. (2014). La formación del profesorado en TIC: Modelo TPACK. Secretariado de Recursos Audiovisuales y Nuevas Tecnologías de la Universidad de Sevilla. Recuperado de https://www.researchgate.net/publication/266733957

- Cabero Almenara, J.; Marín Díaz, V. y Castaño Garrido, C. (2015). Validación de la aplicación del modelo TPACK para la formación del profesorado en TIC. @tic revista d'innovació educativa, 14, pp. 13-22.

- Cejas-León, R., Navío-Gámez, A. y Barroso-Osuna, J. (2016). Las competencias del profesorado universitario desde el modelo TPACK (Conocimiento Tecnológico y Pedagógico del Contenido). Pixel-Bit. Revista de Medios y Educación, 49, pp. 105-119.

- Coll, C. (2011). Aprender y enseñar con las TIC: expectativas, realidad y potencialidades. En R. Carneiro; J. Toscano y T. Díaz (Coord.) Los desafíos de las TIC para el cambio educativo (pp.115-125). España: OEI y Fundación Santillana.

- Coll, C. y Monereo, C. (2008). Psicología de la educación virtual. Madrid: Morata.

- Cruz Tomé, M. (2003). Necesidad y objetivos de la formación pedagógica del profesor universitario. Revista de Educación, 331, pp. 35-66.

- Demuth, P. y Sánchez, E. (2017). El desarrollo del conocimiento docente universitario: lo pedagógico, lo profesional de base y la investigación. Matices de la docencia universitaria experimentada. Praxis Educativa, 21(2), pp. 29-38.

- Ertmer, P. (2005). Teacher pedagogical beliefs: The final frontier in our quest for technology integration? Educational Technology Research and Development, 53(4), pp. 25-39.

- Flores, F.; Ortiz, M. y Buontempo, M. (2018). TPACK: un modelo para analizar prácticas docentes universitarias. El caso de una docente experta. REDU. Revista de Docencia Universitaria, 16(1), pp. 119136.

- Gewerc, A. (2001). Identidad Profesional y trayectoria en la universidad. Profesorado. Revista de curriculum y formación del profesorado, 5, pp.1-15. 
Revista de la Escuela de Ciencias de la Educación, año 15, nRo. 14, vol 2. Julio a Diciembre de 2019. PÁGinas 14-27. ISSN 18516297 (DESDE DICIEMBRE DE 2006 A DICIEMBRE DE 2017). ISSN 2362-3349 (EN LÍNEA). EL MODELO TPACK EN LA PRAXIS DOCENTE EN UNA UNIVERSIDAD ARGENTINA. CONOCIMIENTOS Y PRÁCTICAS DOCENTES EN TORNO AL CONOCIMIENTO DIDÁCTICO-TECNOLÓGICO DEL CONTENIDO (CDTC) en aulas universitarias. Fernando, A. Flores. Margarita, C. Ortiz.

- Gewerc, A.; Pernas, E., y Varela, J. (2013). Conocimiento tecnológico - didáctico del contenido en la enseñanza de ingeniería informática: un estudio de caso colaborativo con la perspectiva del docente y los investigadores. Docencia Universitaria REDU, 11, pp. 349-374.

- Hew, K. y Brush, T. (2007). Integrating technology into K-12 teaching and learning: Current knowledge gaps and recommendations for future research. Educational Technology Research and Development, 55, pp. 223-252.

- Jang, S.-J. \& Tsai, M.-F. (2013). Exploring the TPACK of Taiwanese secondary school science teachers using a new contextualized TPACK model. Australasian Journal of Educational Technology, 29(4), pp. 566-580.

- Koehler, M.; Mishra, P. y Cain, W. (2015). ¿Qué son los saberes tecnológicos y pedagógicos del contenido (TPACK)?. Virtualidad, Educación y Ciencia, 10 (6), pp. 9 -23

- $\quad$ Lindblom-Ylänne, S.; Trigwell, K.; Nevgi, A. y Ashwin, P. (2006). How approaches to teaching are affected by discipline and teaching context. Studies in Higher Education, 31 (3), pp. 285-291.

- Lion, C. (2006). Imaginar con tecnologías. Relaciones entre tecnologías y conocimiento. Buenos Aires: La crujía.

- $\quad$ Litwin, E. (2005). Tecnologías educativas en tiempos de Internet. Buenos Aires: Amorrortu.

- Lueddeke, G. (2003). Professionalising Teaching Practice in Higher Education: a study of disciplinary variation and 'teaching-scholarship'. Studies in Higher Education, 28 (2), pp. 213-228.

- Marcelo, C. (2013). Las tecnologías para la innovación y la práctica docente. Revista Brasileira de Educação, 18(52), pp. 25-47.

- Marcelo, C. y Vaillant, D. (2009). Desarrollo profesional docente: ¿Cómo se aprende a enseñar? Madrid: Narcea.

- Marcelo, C.; Yot, C. y Mayor, C. (2015). Enseñar con tecnologías digitales en la universidad. Comunicar, 23(45), pp. 117-124.

- Marcelo C.; Yot, C. y Perera, R. (2016). El conocimiento tecnológico y tecno-pedagógico en la enseñanza de las ciencias en la universidad. Un estudio descriptivo. Enseñanza de las ciencias, 34(2), pp. 67-86.

- Mishra, P. \& Koehler, M. (2006). Technological pedagogical content knowledge (TPCK): Confronting the wicked problems of teaching with technology. In C. Crawford et al. (Eds.), Proceedings of society for information technology and teacher education international conference, pp. 2214 - 2226. USA: Chesapeake.

- $\quad$ Roig R. y Flores, C. (2014). Conocimiento tecnológico, pedagógico y disciplinario del profesorado: el caso de un centro educativo inteligente. EDUTEC, Revista Electrónica de Tecnología Educativa, 47. Recuperado de http://edutec.rediris.es/Revelec2/Revelec47/n47_Roig-Flores.html

- Roig-Vila, R., Mengual-Andrés, S., \& Quinto-Medrano, P. (2015). Primary Teachers' Technological, Pedagogical and Content Knowledge. Media Education Research Journal, 23(45), pp. 151-159.

- Roig-Vila, R. (2016). Educación y Tecnología. Propuestas desde la Investigación y la Innovación Educativa. Barcelona: Octaedro.

- Sánchez, J. (2003). Integración curricular de las TIC: conceptos e ideas. Revista Enfoques Educacionales, 5(1), pp. 51-65.

- $\quad$ Sandín Esteban, P. (2003). Investigación cualitativa en educación. Fundamentos y tradiciones. Madrid: Mc Graw Hill.

- Schmidt, D.; Baran, E.; Thompson, A.; Mishra, P.; Koehler, M. y Shin, T. (2009). Technological pedagogical content knowledge (TPACK): The development and validation of an assessment instrument for preservice Teachers. Journal of Research on Technology in Education, 42(2), pp. 123-149.

- Shulman, L. (1986). Those who understand: Knowledge growth in teaching. Educational Researcher, 15(2), pp. $4-14$.

- $\quad$ Stake, R. (2007). Investigación con estudios de caso. Madrid: Morata. 